\title{
OPTIMIZACIÓN DE LA GEOMETRÍA DE LA BOMBA HIDRÁULICA TIPO JET
}

\author{
Vinicio Melo ${ }^{1}$
}

\section{RESUMEN}

Este trabajo presenta un método de cálculo directo para determinar la geometría óptima de una bomba jet para una aplicación determinada. Se revisan los conceptos necesarios del bombeo hidráulico tipo jet para poder explicar el procedimiento de dimensionamiento. El método usa las curvas de comportamiento de las bombas jet. Se calcula un área de tobera y se selecciona una relación de áreas. Esta información se usa para seleccionar una de las geometrías dadas por cualquier fabricante. Se presenta la secuencia de cálculos junto con un ejemplo de aplicación.

\section{INTRODUCCIÓN}

El bombeo tipo jet es una variante del bombeo hidráulico, el cual ha incrementado su aplicación debido a su flexibilidad y durabilidad. El principio básico de este sistema de levantamiento artificial, es inyectar un fluido a alta presión hacia el fondo del pozo (fluido motriz), para transferir energía a la bomba de fondo y de esta manera poder operarla. El bombeo hidráulico tipo jet tiene la ventaja de que se puede aplicar en pozos profundos y desviados.

En las bombas de desplazamiento positivo, como en el caso del bombeo hidráulico tipo pistón y del bombeo mecánico, se reduce su vida útil cuando se tienen sólidos presentes, este efecto también se produce en el bombeo electrosumergible. Sin embargo, esto no sucede cuando se utiliza el bombeo hidráulico tipo jet, ya que no tiene partes en movimiento. Además es posible operarlo durante más tiempo en medios corrosivos y fluidos que contienen arena.

\footnotetext{
${ }^{1}$ Facultad de Ciencias de la Ingeniería, Universidad Tecnológica Equinoccial, Av. Occidental y Mariana de Jesús. Quito, Ecuador.mgvr2305@ute.edu.ec
} 
En los artículos publicados por Petrie y otros, se propone un método para calcular la potencia, HP, requerida por una bomba previamente seleccionada, o mediante el funcionamiento de ésta, determinar el comportamiento de afluencia del pozo, IPR. En ambos casos es necesario especificar la geometría de la bomba para efectuar los cálculos.

La solución se obtiene mediante el método de ensaye y error, para seleccionar una bomba en la cual no se tenga cavitación, sin embargo, no enfoca el problema a la selección de la bomba óptima (diámetro de tobera y cámara de mezclado más adecuados), para la operación del sistema.

El método que se presenta en este trabajo es el propuesto por Eddie E. Smart, de la división Guiberson. Con este método es posible calcular directamente la geometría óptima de una bomba tipo Jet, para un conjunto de condiciones dadas.

\section{Aspectos teóricos del bombeo hidráulico tipo jet.}

El principio de operación del bombeo hidráulico tipo jet, se basa en la inyección de un fluido, denominado fluido motriz, hasta la profundidad de la bomba de fondo. Dicho fluido, llega a la tobera a una alta presión definida como $\mathrm{P}_{\mathrm{N}}$, (Fig. 1). En este punto el fluido motriz a alta presión es dirigido a través de la tobera, la cual transforma la energía potencial (presión) en energía cinética (fluido a alta velocidad), disminuyendo considerablemente la presión del fluido motriz.

La baja presión del fluido motriz permite que los fluidos del yacimiento entren al pozo y posteriormente a la bomba de fondo, a la presión $\mathrm{P}_{\mathrm{S}} \mathrm{y}$ a la tasa de producción $\mathrm{Q}_{\mathrm{s}}$. La alta velocidad (momentum) del fluido motriz se mezcla con la baja velocidad (momentum) de los fluidos producidos, en una sección de área constante denominada cámara de mezclado o garganta (throat). A la mezcla de fluidos se le llama fluido de retorno.

Cuando el fluido de retorno, alcanza la parte final de la cámara de mezclado, tiene baja presión y alta velocidad. El fluido entonces sale de la bomba a través del difusor, para transformar la energía cinética en presión, estableciéndose de esta manera nuevamente un estado de alta presión y baja velocidad. Esta alta presión de descarga $\mathrm{P}_{\mathrm{D}}$, debe ser suficiente para llevar la tasa del fluido de retorno $\mathrm{Q}_{\mathrm{D}}$, hasta la superficie. 
Al diseñar el sistema de bombeo hidráulico tipo jet, se deben satisfacer dos condiciones. La primera se refiere a la tasa de fluido que puede bombearse a través de una tobera de diámetro dado, para una determinada caída de presión. Esta condición se expresa mediante la ecuación 1:

$$
Q_{N}=832 A_{N} \sqrt{\frac{P_{N}-P_{S}}{G_{N}}}
$$

La segunda condición se describe mediante las curvas de comportamiento adimensional que relacionan la presión de entrada a la tobera $\mathrm{P}_{\mathrm{N}}$, la presión de succión de los fluidos del pozo $\mathrm{P}_{\mathrm{S}} \mathrm{y}$ la presión de descarga de la bomba $\mathrm{P}_{\mathrm{D}}$, con la tasa que pasa a través de la tobera $\mathrm{Q}_{\mathrm{N}} \mathrm{y}$ la tasa de fluido producido que ingresa a la bomba $Q_{S}$ (Fig. 1). Dichas curvas se muestran en la Fig. 2 y se definen mediante la ecuación 2:

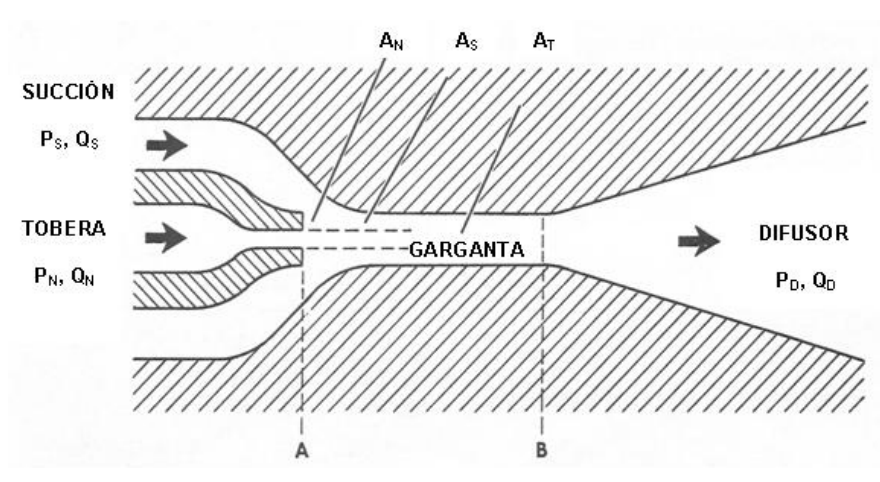

Figura 1. Bombeo hidráulico tipo Jet.

$$
H=\frac{N U M}{\left(1+K_{N}\right)-N U M}
$$

Dónde:

$$
\begin{aligned}
N U M=2 R+(1-2 R)\left[\frac{M \times R}{1-R}\right]^{2}-\left(1+K_{T D}\right) R^{2}(1+M)^{2} \\
R=\frac{A_{N}}{A_{T}} \\
M=\frac{Q_{S} \times G_{S}}{Q_{N} \times G_{N}} \\
H=\frac{P_{D}-P_{S}}{P_{N}-P_{D}}
\end{aligned}
$$


Factores involucrados en la selección de la geometría de la bomba jet

Las ecuaciones 1 y 2 involucran dos áreas, las cuales determinan el comportamiento de la bomba. En la ecuación 1 , el área de la tobera $A_{\mathrm{N}}$ se relaciona con la tasa de fluido motriz requerido $\mathrm{Q}_{\mathrm{N}}$. En la ecuación 2, el valor de $\mathrm{R}$ determina la forma de las curvas de comportamiento adimensional. La ecuación 3, expresa la relación de áreas $\mathrm{R}$, entre el área de la tobera y el área de la cámara de mezclado. Por lo que, dichas áreas son los parámetros a modificar, con la finalidad de encontrar la bomba óptima para las condiciones de producción del pozo.

Si en la ecuación 2 se mantiene constante la relación de áreas $\mathrm{R}$, se puede graficar en una curva la relación de presiones $\mathrm{H}$ vs la relación de flujo adimensional $\mathrm{M}$. Al cambiar el valor de $\mathrm{R}$ se puede generar otra curva como se ilustra en la figura 2. Analizando esta gráfica, se observa que para $\mathrm{R}=0.6 \mathrm{el}$ valor de $\mathrm{H}$ es el más grande, si los valores de $\mathrm{M}$ son menores que 0.18 , ya que en este punto se cruzan las curvas de $\mathrm{R}=0.6$ y $\mathrm{R}=0.5$. A partir de este valor la curva de $\mathrm{R}=0.5$ proporciona los valores de $\mathrm{H}$ más grandes, hasta intersecarse con la de $\mathrm{R}=0.4$, que ocurre para el valor de $\mathrm{M}=0.33$. Este comportamiento continúa a medida que el valor de $\mathrm{R}$ disminuye.

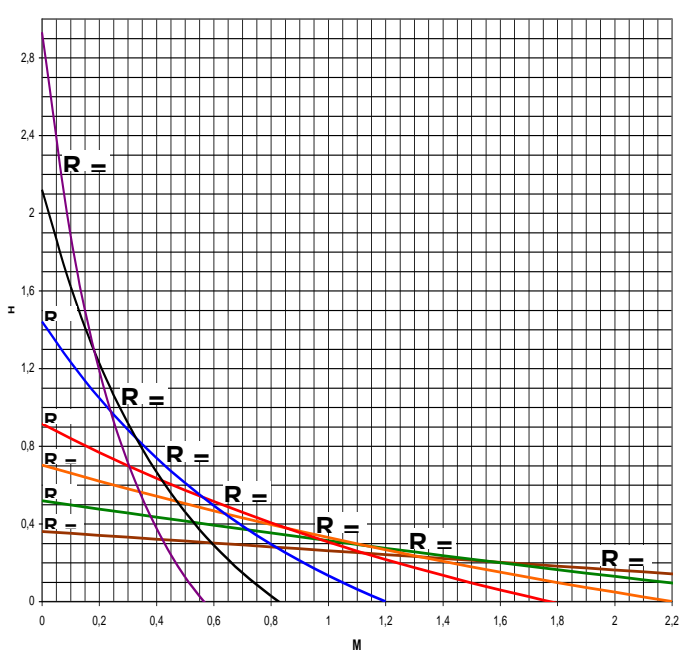

Figura 2. Curvas $\mathrm{H}-\mathrm{M}$ de Guiberson

La ecuación 5 representa la relación de presiones, $\mathrm{H}$, la cual se puede resolver para $\mathrm{P}_{\mathrm{N}}$ de la siguiente manera: 


$$
P_{N}=\frac{P_{D}-P_{S}}{H}+P_{D}
$$

El término $\mathrm{P}_{\mathrm{N}}$ es una combinación de la presión de operación superficial, la presión hidrostática del fluido motriz y las pérdidas de presión del fluido motriz en la tubería.

En la ecuación 6 se puede observar que si permanecen constantes las presiones $\mathrm{P}_{\mathrm{S}}$ y $\mathrm{P}_{\mathrm{D}}$, a medida que el valor de $\mathrm{H}$ aumenta, se reduce la presión $\mathrm{P}_{\mathrm{N}}$. Esto repercutiría en bajos valores de la presión de operación superficial y bajos requerimientos de potencia.

Si se relaciona esta observación con la Fig. 2, esto significa que para un valor dado de M, la curva que proporcionará la menor presión de operación será aquella que tenga el más alto valor de $\mathrm{H}$. Con base en esto, se podría usar una Curva de Comportamiento de Diseño como la mostrada en la Fig. 3, compuesta por los segmentos de línea que representan los máximos valores de $\mathrm{H}$, en las curvas de la Fig. 2. Es decir, que la Curva de Comportamiento de Diseño es la envolvente superior de las curvas de la Fig. 2.

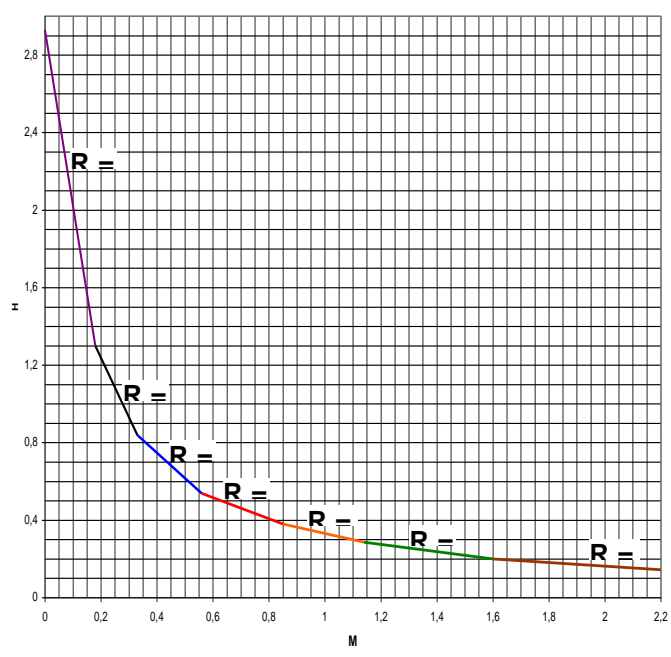

Figura 3. Curva de comportamiento de diseño Guiberson

La Curva de Comportamiento de Diseño se puede utilizar como si fuera la curva de comportamiento de una sola bomba, para calcular la relación de presiones $\mathrm{H}$ y la relación de flujo adimensional $\mathrm{M}$, que sea consistente con la descripción del pozo y la curva de IPR. Para calcular la geometría óptima de la bomba se debe especificar la presión de operación superficial deseada. Como regla general, la mayor 
eficiencia se obtiene con la mayor presión de operación superficial, debido a que se requiere una menor tasa de fluido motriz y como consecuencia se tiene menos pérdidas de presión por fricción en la tubería.

Tabla 1. Relaciones de áreas óptimas

\begin{tabular}{cc}
\hline RELACIÓN DE & RANGO DE RELACIÓN DE \\
\hline 0.60 & $2.930-1.300$ \\
0.50 & $1.300-0.839$ \\
0.40 & $0.839-0.538$ \\
0.30 & $0.538-0.380$ \\
0.25 & $0.380-0.286$ \\
0.20 & $0.286-0.160$ \\
0.15 & 0.160 \\
\hline
\end{tabular}

Los valores mostrados en la tabla 1 son para los puntos de intersección de las curvas indicadas en la figura 2. Una tabla similar puede ser construida para otros fabricantes determinando los puntos donde se intersecan las Curvas de Comportamiento.

Para los valores de $\mathrm{M}$ y $\mathrm{H}$ obtenidos habrá una relación de áreas, $\mathrm{R}$, de la Curva de Comportamiento de Diseño correspondiente a esos valores. Debido a que ésta es la solución para una tasa de producción y una presión de fondo fluyente dadas, entonces el valor de M junto con el de la producción deseada, se pueden utilizar para calcular la tasa de fluido motriz, así como la presión de fondo de éste a la entrada de la tobera, $\mathrm{P}_{\mathrm{N}}$. Con esta información se podrá utilizar la ecuación (1) para calcular el área exacta de la tobera, para que la tasa obtenida del fluido motriz pase a través de ella.

El objetivo al seleccionar la geometría óptima de la bomba tipo jet es: primero, escoger la bomba que levante el fluido con los menores requerimientos de potencia y, segundo, que no exista cavitación en la bomba. La cavitación ocurriría en la bomba cuando la presión estática del fluido producido dentro de la cámara de mezclado sea menor que la presión de saturación del fluido producido. Al ocurrir la cavitación la cámara de mezclado puede resultar dañada, por lo que es necesario seleccionar otra bomba, la cual aunque requiera mayor potencia HP, evitaría dichos daños. 
Los límites para la cavitación en la bomba se pueden predecir mediante modelos matemáticos teóricos o utilizando pruebas de laboratorio para establecer las constantes de dichas ecuaciones teóricas. La ecuación (7) representa la relación de flujo adimensional en el límite de la cavitación. Cuando la relación de flujo adimensional es mayor que la relación de flujo adimensional en el límite de cavitación, esta puede provocar daño a la bomba.

Ecuación de la relación de flujo adimensional en el límite de cavitación:

$$
M_{L}=\frac{(1-R)}{R} \sqrt{\frac{P_{S}}{1.3\left(P_{N}-P_{S}\right)}}
$$

\section{$\underline{\text { Secuencia de cálculo }}$}

A continuación se presenta la secuencia de cálculo propuesta por Smart para determinar la geometría óptima de la bomba jet.

1. Fijar la presión de operación superficial deseada, $\mathrm{P}_{\mathrm{T}}$.

2. Como valor inicial suponer una relación de flujo adimensional igual a 1. Este es utilizado únicamente para calcular las pérdidas de presión por fricciones iniciales.

3. Calcular el gradiente de presión del petróleo producido a partir de su gravedad API.

$$
G_{O}=\frac{0.433 \times 141.5}{131.5+{ }^{\circ} A P I}
$$

4. Calcular el gradiente de presión del fluido producido, basado en los gradientes de petróleo y agua.

$$
G_{S}=F_{W} \times G_{W}+F_{O} \times G_{O}
$$

Donde: $F_{O}=1-F_{W}$

5. Estimar el factor de volumen de formación para el petróleo y el agua.

$$
B_{T}=\left[1+2.8\left(\frac{G O R}{P_{S}}\right)^{1.2}\right] F_{O}+F_{W}
$$


6. Calcular la tasa del fluido motriz, con base en la producción deseada y la relación de flujo adimensional, $\mathrm{M}$.

$$
Q_{N}=\frac{G_{S} \times Q_{S} \times B_{T}}{G_{N} \times M}
$$

$G_{N}=$ Gradiente de fluido motriz que pasa a través de la tobera.

7. Utilizando la ecuación:

$$
P_{F}=\left[\frac{2.02 \times 10^{-6} \times L \times\left(\left(D_{1}+D_{2}\right) \times \mu\right)^{0.21}}{C \times G^{0.21}}\right] G \times Q^{1.79}
$$

Dónde:

\begin{tabular}{ccc} 
& $C=\left(D_{1}-D_{2}\right)\left(D_{1}{ }^{2}-D_{2}{ }^{2}\right)^{2}$ & $\left(D_{1} /\left(D_{1}-D_{2}\right)\right)^{0.1}$ \\
\hline & Flujo anular & Flujo por T.P. \\
\hline $\mathrm{D}_{1}$ & $\mathrm{D}_{\text {iTR }}$ & $\mathrm{D}_{\text {iTP }}$ \\
$\mathrm{D}_{2}$ & $\mathrm{D}_{\text {oTP }}$ & 0 \\
\hline
\end{tabular}

Calcular las pérdidas de presión por fricción en la tubería por la que fluye el fluido motriz, ya sea a través de una sección anular o circula, y considerar que:

$\mathrm{P}_{\mathrm{FN}}=$ pérdida de presión por fricción del fluido motriz.

$\mathrm{P}_{\mathrm{FD}}=$ pérdida de presión por fricción del fluido de retorno.

8. Calcular la presión del fluido motriz en la tobera $\mathrm{P}_{\mathrm{N}}$, como la suma de la presión de operación más la presión hidrostática del fluido motriz, menos la pérdida de presión por fricción de éste, en la tubería.

$$
P_{N}=P_{T}+G_{N} \times D-P_{F N}
$$

9. Calcular la tasa del fluido de retorno $\mathrm{Q}_{\mathrm{D}}$, como la suma de la tasa de producción y la tasa del fluido motriz.

$$
Q_{D}=Q_{N}+Q_{S}
$$

10. Calcular el gradiente del fluido de retorno $G_{D}$, como un promedio ponderado del gradiente del fluido motriz y el gradiente del fluido producido. 


$$
G_{D}=\frac{G_{S} \times Q_{S}+G_{N} \times Q_{N}}{Q_{D}}
$$

11. Calcular la fracción de agua del fluido de retorno $\mathrm{F}_{\mathrm{WD}}$, dependiendo si el fluido motriz es petróleo o agua, con las siguientes ecuaciones:

- Si el fluido motriz es petróleo:

$$
F_{W D}=\frac{Q_{S} \times F_{W}}{Q_{D}}
$$

- Si el fluido motriz es agua:

$$
F_{W D}=\frac{Q_{N}+Q_{S} \times F_{W}}{Q_{D}}
$$

12. Determinar la relación gas - líquido del fluido de retorno GLR.

$$
G L R=\frac{Q_{S} \times F_{O} \times G O R}{Q_{D}}
$$

13. Determinar la viscosidad del fluido de retorno $\mu_{\mathrm{D}}$, como un promedio ponderado de las viscosidades del agua y del petróleo.

$$
\mu_{D}=F_{W D} \times \mu_{W}+\left(1-F_{W D}\right) \times \mu_{O}
$$

14. Determinar la presión de descarga de la bomba $\mathrm{P}_{\mathrm{D}}$, como la suma de la presión hidrostática del fluido de retorno, la caída de presión por fricción en el conducto de retorno y la contrapresión en la cabeza del pozo. Si la GLR es menor que $10 \mathrm{pie}^{3} / \mathrm{bl}$, determinar $\mathrm{P}_{\mathrm{FD}}$ con la ecuación 12.

$$
P_{D}=P_{W H}+G_{D} \times D+P_{F D}
$$

Si la GLR es mayor o igual que $10 \mathrm{pie}^{3} / \mathrm{bl}$, se debe utilizar una correlación adecuada para flujo multifásico.

15. Calcular un nuevo valor de la relación de presiones $H$, mediante la ecuación 5 .

16. Basado en este valor de $\mathrm{H}$ y la Fig. 3 o la tabla 1, se determina la relación de áreas óptima, R.

17. Utilizando la Curva de Comportamiento de Diseño de la Fig. 3, se encuentra un nuevo valor para M correspondiente al valor de $\mathrm{H}$ del paso 15. También se puede utilizar la siguiente ecuación para calcular M, usando el valor de $\mathrm{R}$ obtenido en el paso anterior. 


$$
M=\frac{C_{3}-\sqrt{C_{2} \times C_{3}+C_{3} \times C_{1}-C_{2} \times C_{1}+\frac{C_{4}\left(C_{2}-C_{3}\right) H}{H+1}}}{C_{2}-C_{3}}
$$

Dónde:

$$
\begin{array}{ll}
C_{1}=2 R & K_{T D}=0.20 \\
C_{2}=\frac{(1-2 R) R^{2}}{(1-R)^{2}} & K_{N}=0.03 \\
C_{3}=\left(1+K_{T D}\right) R^{2} & \\
C_{4}=1+K_{N} &
\end{array}
$$

Si en el paso No. 20 se determina la existencia de cavitación, se recomienda usar las Curvas de Comportamiento de la Fig. 2, para encontrar un nuevo valor de $\mathrm{M}$ en lugar de la Fig. 3. Usar el valor de R determinado en el paso 16. En vez de usar la Fig. 3 se puede utilizar la ecuación 20.

18. Comparar el nuevo valor de $\mathrm{M}$ con el anterior, si la variación de $\mathrm{M}$ es menor del 1\%, se considera que se ha obtenido la convergencia y se continúa en el paso 19. Caso contrario regresar al paso 6 usando el nuevo valor de $\mathrm{M}$.

19. Calcular la relación de flujo adimensional en el límite de cavitación, $\mathrm{M}_{\mathrm{L}}$, con la ecuación 7 .

20. Si $\mathrm{M}<\mathrm{M}_{\mathrm{L}}$, no existe problema de cavitación, en tal caso continuar en el paso 24. Si $\mathrm{M}>\mathrm{M}_{\mathrm{L}}$, entonces se tendrán problemas de cavitación, por lo que se requiere un ajuste y continuar en el paso siguiente.

21. Fijar $\mathrm{M}=\mathrm{M}_{\mathrm{L}}$ y utilizar el valor de la relación de áreas seleccionada para calcular un nuevo valor de la relación de presiones H. La curva de comportamiento de la Fig. 2 también se puede usar para encontrar el valor de $\mathrm{H}$ correspondiente a $\mathrm{M}_{\mathrm{L}}$. El valor de $\mathrm{R}$ se debe mantener constante en los cálculos para evitar cavitación.

22. Se calcula la presión de operación superficial requerida para evitar la cavitación:

$$
P_{T}=\frac{P_{D}-P_{S}}{H}+P_{D}-G_{N} \times D+P_{F N}
$$

23. Repetir los cálculos para evitar cavitación, regresando al paso 5.

24. Determinar el área de la tobera requerida para manejar la tasa de fluido motriz calculada en el paso 6, despejando $\mathrm{A}_{\mathrm{N}}$ de la ecuación 1. 


$$
A_{N}=\frac{Q_{N}}{832 \sqrt{\frac{P_{N}-P_{S}}{G_{N}}}}
$$

La relación de áreas encontrada en el paso 16 junto con el área de la tobera del paso 24 definen la geometría óptima de la bomba tipo jet, para la presión de operación superficial dada. Esta área de la tobera es la medida ideal requerida para que la tasa calculada del fluido motriz pase a través de ella. Generalmente el diámetro exacto de la tobera no es el comercial y no se encuentra disponible, por lo que se selecciona el diámetro disponible más cercano, así como la cámara de mezclado que combina con esta tobera comercialmente disponible, para obtener la relación de áreas óptima.

\section{Ejemplo de aplicación:}

Con el fin de ilustrar el procedimiento de cálculo anterior se presentan los siguientes datos de un pozo:

Profundidad

Longitud de la T.P.

Diámetro exterior de la T.P.

Diámetro interior de la T.P.

Diámetro interior de la tubería de retorno

Presión en la cabeza del pozo

Fluido motriz

Densidad del petróleo

Gradiente del agua

Viscosidad del petróleo

Viscosidad del agua

Relación gas en solución - petróleo

Fracción de agua

Tasa de producción

$$
\mathrm{D}=5000 \text { pies }
$$

$\mathrm{L}=6000$ pies

$\mathrm{D}_{\text {отP }}=2.375 \mathrm{plg}$

$\mathrm{D}_{\text {iTP }}=1.995 \mathrm{plg}$

$\mathrm{D}_{\text {iTR }}=4.892 \mathrm{plg}$

$\mathrm{P}_{\mathrm{WH}}=100 \mathrm{lb} / \mathrm{plg}^{2}$

Petróleo

$30^{\circ} \mathrm{API}$

$\mathrm{G}_{\mathrm{W}}=0.45 \mathrm{lb} / \mathrm{pg}^{2} / \mathrm{pie}$

$\mu_{\mathrm{O}}=2.5 \mathrm{cp}$

$\mu_{\mathrm{W}}=0.55 \mathrm{cp}$

$\mathrm{GOR}=0 \mathrm{pie}^{3} / \mathrm{bl}$

$\mathrm{F}_{\mathrm{W}}=0.3$

$\mathrm{Q}_{\mathrm{s}}=500 \mathrm{bl} / \mathrm{día}$ 


\section{Solución:}

1. Fijar la presión de operación superficial deseada, $P_{T}$.

$$
P_{T}=3000 \mathrm{lb} / \mathrm{pg}^{2}
$$

2. Como valor inicial suponer una relación de flujo adimensional igual a 1. Este es utilizado únicamente para calcular las pérdidas de presión por fricciones iniciales.

$$
M=1
$$

3. Calcular el gradiente de presión del petróleo producido a partir de su gravedad API.

$$
G_{O}=\frac{0.433 \times 141.5}{131.5+{ }^{\circ} \mathrm{API}}
$$

$$
G_{O}=\frac{0.433 \times 141.5}{131.5+30}=0.3794 \mathrm{lb} / \mathrm{pg}^{2} / \mathrm{pie}
$$

4. Calcular el gradiente de presión del fluido producido, basado en los gradientes de petróleo y agua.

$G_{S}=F_{w} \times G_{w}+F_{O} \times G_{O}$

$F_{O}=1-0.3=0.7$

$G_{S}=0.3 \times 0.45+0.7 \times 0.3794$

$G_{S}=0.4006 \mathrm{lb} / \mathrm{pg}^{2} / \mathrm{pie}$

5. Estimar el factor de volumen de formación, para el petróleo y el agua.

$$
B_{T}=\left[1+2.8\left(\frac{G O R}{P_{S}}\right)^{1.2}\right] F_{O}+F_{W} B_{T}=\left[1+2.8\left(\frac{0}{1000}\right)^{1.2}\right](0.7)+0.3
$$

6. Calcular la tasa del fluido motriz, con base en la producción deseada y la relación de flujo adimensional, $\mathrm{M}$. 
$Q_{N}=\frac{G_{S} \times Q_{S} \times B_{T}}{G_{N} \times M}$

$Q_{N}=\frac{0.4006 \times 500 \times 1}{0.3794 \times 1}=527.9229 \mathrm{bl} / \mathrm{dí}$

$G_{N}=0.3794 \mathrm{lb} / \mathrm{pg}^{2} /$ pie ya que el fluido motriz es petróleo

7. Utilizando la ecuación (12) calcular las caídas de presión del fluido motriz en la tubería.

$$
\begin{aligned}
& P_{F}=\left[\frac{2.02 \times 10^{-6} \times L \times\left(\left(D_{1}+D_{2}\right) \times \mu\right)^{0.21}}{C \times G^{0.21}}\right] G \times Q^{1.79} \\
& C=\left(D_{1}-D_{2}\right)\left(D_{1}^{2}-D_{2}^{2}\right)^{2}\left(D_{1} /\left(D_{1}-D_{2}\right)\right)^{0.1} \\
& D_{1}=1.995 \mathrm{pg}, \\
& D_{2}=0, L=6000 \mathrm{pies},
\end{aligned}
$$

8. Calcular la presión del fluido motriz en la tobera $P_{N}$, como la suma de la presión de operación más la presión hidrostática del fluido motriz, menos la pérdida de presión por fricción de éste, en la tubería.

$P_{N}=P_{T}+G_{N} \times D-P_{F N}$

$P_{N}=3000+0.3794 \times 5000-18.6729$

$P_{N}=4878.2157 \mathrm{lb} / \mathrm{pg}^{2}$

9. Calcular la tasa del fluido de retorno $Q_{D}$, como la suma de la tasa producida y la tasa del fluido motriz.

$Q_{D}=Q_{N}+Q_{S} Q_{D}=527.9229+500=1027.9229 \mathrm{bl} / \mathrm{dí} \mathrm{a}$

10. Calcular el gradiente del fluido de retorno $G_{D}$, como un promedio ponderado del gradiente del fluido motriz y el gradiente del fluido producido. 
$G_{D}=\frac{G_{S} \times Q_{S}+G_{N} \times Q_{N}}{Q_{D}}$

$$
\begin{aligned}
& G_{D}=\frac{0.4006 \times 500+0.3794 \times 527.9229}{1027.9229} \\
& G_{D}=0.3897 \mathrm{lb} / \mathrm{pg}^{2} / \mathrm{pie}
\end{aligned}
$$

11. Calcular la fracción de agua del fluido de retorno $F_{W D}$, considerando que el fluido motriz es petróleo.

$$
\begin{aligned}
& F_{W D}=\frac{Q_{S} \times F_{W}}{Q_{D}} \\
& F_{W D}=\frac{500 \times 0.3}{1027.9229}=0.1459
\end{aligned}
$$

12. Determinar la relación gas líquido del fluido de retorno GLR.

$$
G L R=\frac{Q_{S} \times F_{O} \times G O R}{Q_{D}}
$$

$$
G L R=\frac{500 \times 0.7 \times 0}{1027.9229}=0 \mathrm{pie}^{3} / \mathrm{bl}
$$

13. Determinar la viscosidad del fluido de retorno $\mu_{\mathrm{D}}$, como un promedio ponderado de las viscosidades del agua y del petróleo.

$$
\mu_{D}=F_{W D} \times \mu_{W}+\left(1-F_{W D}\right) \times \mu_{O} \begin{aligned}
& \mu_{D}=0.1459 \times 0.55+(1-0.1459) \times 2.5 \\
& \mu_{D}=2.2154 c p
\end{aligned}
$$

14. Determinar la presión de descarga de la bomba $P_{D}$, como la suma de la presión hidrostática del fluido de retorno, la caída de presión por fricción en el conducto de retorno y la contrapresión en la cabeza del pozo. Si GLR es menor que $10 \mathrm{pie}^{3} / \mathrm{bl}$, determinar $P_{F D}$ con la ecuación (12).

$$
\begin{aligned}
& P_{D}=P_{W H}+G_{D} \times D+P_{F D} P_{F}=\left[\frac{2.02 \times 10^{-6} \times L \times\left(\left(D_{1}+D_{2}\right) \times \mu\right)^{0.21}}{C \times G^{0.21}}\right] G \times Q^{1.79} \\
& C=\left(D_{1}-D_{2}\right)\left(D_{1}^{2}-D_{2}^{2}\right)^{2}\left(D_{1} /\left(D_{1}-D_{2}\right)\right)^{0.1} \\
& D_{1}=4.892 \mathrm{pg}, \quad D_{2}=2.375 \mathrm{pg}, \mathrm{L}=6000 \mathrm{pies}, \mu_{D}=2.2154 \mathrm{cp} \\
& G_{D}=0.3897 \mathrm{lb} / \mathrm{pg}^{2} / \mathrm{pie}, \quad Q_{D}=1027.9229 \mathrm{bl} / \text { dia } \quad P_{F D}=2.8235 \mathrm{lb} / \mathrm{pg}^{2}
\end{aligned}
$$


15. Calcular un nuevo valor de la relación de presiones $\mathrm{H}$, mediante la ecuación (5).

$$
H=\frac{P_{D}-P_{S}}{P_{N}-P_{D}}
$$

$$
H=\frac{2051.2399-1000}{4878.2157-2051.2399}=0.3719
$$

16. Basado en este valor de $\mathrm{H}$ y la Fig. 3 o tabla 1, se determina la relación de áreas óptima R.

De la tabla $1, \mathrm{R}=0.25$

17. Utilizando la Curva de Comportamiento de Diseño Fig. 3, se encuentra un nuevo valor para M correspondiente al valor de $\mathrm{H}$ del paso 15. También se puede utilizar la siguiente ecuación para calcular M, usando el valor de $\mathrm{R}$ obtenido en el paso anterior.

$$
M=\frac{C_{3}-\sqrt{C_{2} \times C_{3}+C_{3} \times C_{1}-C_{2} \times C_{1}+\frac{C_{4}\left(C_{2}-C_{3}\right) H}{H+1}}}{C_{2}-C_{3}}
$$

Dónde:

$$
\begin{array}{lll}
K_{T D}=0.2, & K_{N}=0.03, & H=0.3719 \\
C_{1}=0.5, & C_{2}=0.0556, & C_{3}=0.075,
\end{array} \quad C_{4}=1.03
$$

18. Comparar el nuevo valor de $M$ con el anterior, si la variación de $M$ es menor del $1 \%$, se considera que se ha obtenido la convergencia y se continúa en el paso 19. En caso contrario regresar al paso 6 usando el nuevo valor de $M$.

$M=0.8732, M$ anterior $=1$

No hay convergencia aún, ir al paso 6 .

Segunda iteración: 
6. Calcular la tasa del fluido motriz, con base en la producción deseada y la relación de flujo adimensional, $\mathrm{M}$.

$Q_{N}=\frac{G_{S} \times Q_{S} \times B_{T}}{G_{N} \times M} \quad Q_{N}=\frac{0.4006 \times 500 \times 1}{0.3794 \times 0.8732}=604.5866 \mathrm{bl} / \mathrm{di} a$

$G_{N}=0.3794 \mathrm{lb} / \mathrm{pg}^{2} /$ pie ya que el fluido motriz es petróleo

7. Utilizando la ecuación 12 calcular las caídas de presión del fluido motriz en la tubería.

$P_{F}=\left[\frac{2.02 \times 10^{-6} \times L \times\left(\left(D_{1}+D_{2}\right) \times \mu\right)^{0.21}}{C \times G^{0.21}}\right] G \times Q^{1.79}$

$C=\left(D_{1}-D_{2}\right)\left(D_{1}^{2}-D_{2}^{2}\right)^{2}\left(D_{1} /\left(D_{1}-D_{2}\right)\right)^{0.1}$

$D_{1}=1.995 \mathrm{pg}, \quad D_{2}=0, L=6000$ pies, $\quad \mu_{0}=2.5 \mathrm{cp}$

$G_{N}=0.3794 \mathrm{lb} / \mathrm{pg}^{2} / \mathrm{pie}, \quad Q_{N}=604.5866 \mathrm{bl} / \mathrm{dia} \quad P_{F N}=23.8024 \mathrm{lb} / \mathrm{pg}^{2}$

8. Calcular la presión del fluido motriz en la tobera $P_{N}$, como la suma de la presión de operación más la presión hidrostática del fluido motriz, menos las pérdidas de presión por fricción de éste, en la tubería.

$P_{N}=P_{T}+G_{N} \times D-P_{F N}$

$P_{N}=3000+0.3794 \times 5000-23.8024$

$P_{N}=4873.0861 \mathrm{lb} / \mathrm{pg}^{2}$

9. Calcular la tasa del fluido de retorno $\mathrm{Q}_{\mathrm{D}}$, como la suma de la tasa producida y la tasa del fluido motriz

$Q_{D}=Q_{N}+Q_{S} Q_{D}=604.5866+500=1104.5866 \quad b l / d i ́ a$

10. Calcular el gradiente del fluido de retorno $G_{D}$, como un promedio ponderado del gradiente del fluido motriz y el gradiente del fluido producido.

$$
G_{D}=\frac{G_{S} \times Q_{S}+G_{N} \times Q_{N}}{Q_{D}}
$$

$$
\begin{aligned}
& G_{D}=\frac{0.4006 \times 500+0.3794 \times 604.5866}{1104.5866} \\
& G_{D}=0.3890 \mathrm{lb} / \mathrm{pg}^{2} / \mathrm{pie}
\end{aligned}
$$


11. Calcular la fracción de agua del fluido de retorno $\mathrm{F}_{\mathrm{WD}}$, considerando que el fluido motriz es petróleo.

$$
F_{W D}=\frac{Q_{S} \times F_{W}}{Q_{D}} \quad F_{W D}=\frac{500 \times 0.3}{1104.5866}=0.1358
$$

12. Determinar la relación gas líquido del fluido de retorno GLR.

$$
G L R=\frac{Q_{S} \times F_{O} \times G O R}{Q_{D}} \quad G L R=\frac{500 \times 0.7 \times 0}{1104.5866}=0 \mathrm{pie}^{3} / \mathrm{bl}
$$

13. Determinar la viscosidad del fluido de retorno $\mu_{\mathrm{D}}$, como un promedio ponderado de las viscosidades del agua y del petróleo.

$$
\begin{aligned}
& \mu_{D}=F_{W D} \times \mu_{W}+\left(1-F_{W D}\right) \times \mu_{O} \\
& \mu_{D}=0.1358 \times 0.55+(1-0.1358) \times 2.5 \\
& \mu_{D}=2.2352 c p
\end{aligned}
$$

14. Determinar la presión de descarga de la bomba $P_{D}$, como la suma de la presión hidrostática del fluido de retorno, la caída de presión por fricción en el conducto de retorno y la contrapresión en la cabeza del pozo. Si GLR es menor que $10 \mathrm{pie}^{3} / \mathrm{bl}$, determinar $\mathrm{P}_{\mathrm{FD}}$ con la ecuación 12.

$P_{D}=P_{W H}+G_{D} \times D+P_{F D}$

$P_{F}=\left[\frac{2.02 \times 10^{-6} \times L \times\left(\left(D_{1}+D_{2}\right) \times \mu\right)^{0.21}}{C \times G^{0.21}}\right] G \times Q^{1.79}$

$C=\left(D_{1}-D_{2}\right)\left(D_{1}^{2}-D_{2}^{2}\right)^{2}\left(D_{1} /\left(D_{1}-D_{2}\right)\right)^{0.1}$

$$
\begin{array}{ll}
D_{1}=4.892 \mathrm{pg}, & D_{2}=2.375 \mathrm{pg}, \quad L=6000 \mathrm{pies}, \quad \mu_{D}=2.2352 \mathrm{cp} \\
G_{D}=0.3890 \mathrm{lb} / \mathrm{pg}^{2} / \mathrm{pie}, & Q_{D}=1104.5866 \mathrm{bl} / \mathrm{dia} P_{F D}=3.2128 \mathrm{lb} / \mathrm{pg}^{2} \\
P_{D}=100+0.3890 \times 5000+3.2128 \\
P_{D}=2048.0530 \mathrm{lb} / \mathrm{pg}^{2}
\end{array}
$$


15. Calcular un nuevo valor de la relación de presiones $\mathrm{H}$, mediante la ecuación 5 .

$$
H=\frac{P_{D}-P_{S}}{P_{N}-P_{D}}
$$

$$
H=\frac{2048.0530-1000}{4873.0861-2048.0530}=0.3710
$$

16. Basado en este valor de $\mathrm{H}$ y la Fig. 3 o tabla 1, se determina la relación de áreas óptima R.

De la tabla $1, \mathrm{R}=0.25$

17. Utilizando la Curva de Comportamiento de Diseño de la Fig. 3, se encuentra un nuevo valor para M correspondiente al valor de $\mathrm{H}$ del paso 15. También se puede utilizar la siguiente ecuación para calcular M, usando el valor de R obtenido en el paso anterior.

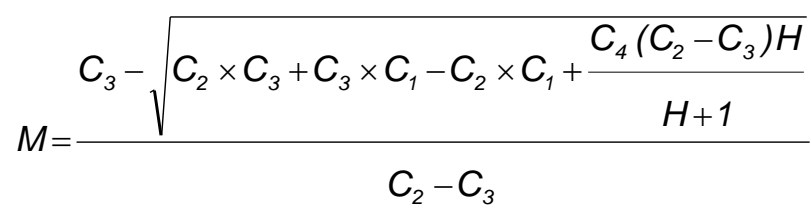

Dónde:

$$
\begin{array}{lll}
\mathrm{K}_{\mathrm{TD}}=0.2, & \mathrm{~K}_{\mathrm{N}}=0.03, & \mathrm{H}=0.3710 \\
\mathrm{C}_{1}=0.5, & \mathrm{C}_{2}=0.0556, & \mathrm{C}_{3}=0.075,
\end{array}
$$

18. Comparar el nuevo valor de $\mathrm{M}$ con el anterior, si la variación de $\mathrm{M}$ es menor del 1\%, se considera que se ha obtenido la convergencia y se continúa en el paso 19. Caso contrario regresar al paso 6 usando el nuevo valor de $\mathrm{M}$.

$M=0.8758, M$ anterior $=0.8732$

$\%$ error $=\left|\frac{0.8758-0.8732}{0.8732}\right| \times 100=0.2970 \%<1 \%$ Por lo tanto se obtuvo la convergencia 
19. Calcular la relación de flujo adimensional en el límite de cavitación, $\mathrm{M}_{\mathrm{L}}$, con la ecuación (7).

$$
M_{L}=\frac{(1-R)}{R} \sqrt{\frac{P_{S}}{1.3\left(P_{N}-P_{S}\right)}} \quad \begin{aligned}
& M_{L}=\frac{(1-0.25)}{0.25} \sqrt{\frac{1000}{1.3(4873.0861-1000)}} \\
& M_{L}=1.3370
\end{aligned}
$$

20. Si $\mathrm{M}<\mathrm{M}_{\mathrm{L}}$, no existe problema de cavitación, en tal caso continuar en el paso 24. Si $\mathrm{M}>\mathrm{M}_{\mathrm{L}}$, entonces se tendrán problemas de cavitación, por lo que se requiere un ajuste y se debe continuar en el paso 21.

$M=0.8758 \quad M_{L}=1.3370$

Por lo tanto, no existen problemas de cavitación. Ir al paso 24.

24. Determinar el área de la tobera requerida, para manejar la tasa del fluido motriz calculada en el paso 6. Utilizando la ecuación 1.

$$
A_{N}=\frac{Q_{N}}{823 \sqrt{\frac{P_{N}-P_{S}}{G_{N}}}}
$$

$$
A_{N}=\frac{604.5866}{823 \sqrt{\frac{4873.0861-1000}{0.3794}}}=0.0072 p^{2}
$$

$R=\frac{A_{N}}{A_{T}}$

$A_{T}=0.0288 \mathrm{pg}^{2}$ Cámara de mezclado. 


\section{RESULTADOS}

La bomba tipo jet que requiere este pozo para producir una tasa de petróleo de $500 \mathrm{bl} /$ día con una presión de operación superficial de $3000 \mathrm{lb} / \mathrm{pg}^{2}$, debe tener un área de tobera de $0.0072 \mathrm{pg}^{2}$, e inyectar una tasa de fluido motriz de $604 \mathrm{bl} /$ día. La curva de comportamiento que tendrá el valor más alto de la relación de presiones $\mathrm{H}$, para estas condiciones se corresponde con una relación de áreas $\mathrm{R}=0.25$. Esto significa que el área de la cámara de mezclado necesita ser cuatro veces más grande que el área de la tobera, o sea $0.0288 \mathrm{pg}^{2}$.

De la tabla de especificaciones de Guiberson se puede observar que no existe una tobera con área de $0.0072 \mathrm{pg}^{2}$. La más cercanas son una tobera A de $0.0055 \mathrm{pg}^{2}$ y una tobera B con área de $0.0095 \mathrm{pg}^{2}$.

Con la tobera A, la cámara de mezclado número 2 proporcionará una relación de áreas de 0.29 y la cámara de mezclado número 3 una relación de áreas de 0.23 . Con la tobera $\mathrm{B}$, la cámara de mezclado número 5 dará una relación de áreas de 0.25 .

Si se fija una presión de operación superficial diferente a la usada en este ejemplo $\left(3000 \mathrm{lb} / \mathrm{pg}^{2}\right), \mathrm{la}^{\mathrm{a}}$ geometría de la bomba tipo jet se modificará. Para una presión de operación menor se obtendrá un mayor diámetro de tobera, mientras que para una presión de operación mayor, el diámetro de la tobera será más pequeño.

Si en el ejemplo anterior se fija una presión de operación de $2500 \mathrm{lb} / \mathrm{pg}^{2}$, el área de la tobera será de $0.0093 \mathrm{pg}^{2}$, con una relación de áreas $\mathrm{R}=0.3$. En este caso la bomba B con cámara de mezclado 4 será la más adecuada (bomba Guiberson B - 4).

Si se hubiera fijado una presión de operación, $\mathrm{P}_{\mathrm{T}}$, mayor a $3000 \mathrm{lb} / \mathrm{pg}^{2}$, habría resultado más adecuado utilizar una tobera A. 


\section{CONCLUSIONES}

La potencia proporcionada por una bomba hidráulica está en función de la presión y de la tasa de fluido motriz suministrada a la bomba. Cuando se diseña una bomba para un pozo específico, se hace un intercambio entre la presión y la tasa. En el caso de la bomba jet este intercambio se cumple moviéndose a lo largo de las Curvas de Comportamiento de la figura 2 o en la Curva de Comportamiento de Diseño de la figura 3. Cuando se incrementa la tasa de fluido motriz, la presión de operación tiende a disminuir de acuerdo a las características de la bomba. Sin embargo, como un resultado de las pérdidas de presión en las tuberías, este incremento en la tasa de fluido motriz tenderá a incrementar la presión de operación. En la mayoría de instalaciones, ya sean las características de la bomba o las pérdidas de presión serán significativamente dominantes las unas sobre las otras.

Si las pérdidas de presión dominan la relación entre presión y tasa, este método usualmente no convergerá para valores razonables de presión de operación. Entonces se requiere un método de ensaye y error para obtener una geometría de bomba adecuada. En algunos casos, el cálculo directo converge para presiones de operación altas y los resultados se usan como valores iniciales para el proceso de ensaye y error. Cuando el comportamiento de la bomba está dominado por las pérdidas de presión por fricción, este método para seleccionar la geometría de la bomba jet es efectivo. 


\section{GLOSARIO}

\section{SÍMBOLO DEFINICIÓN}

$\mathrm{A}_{\mathrm{N}} \quad$ Área de flujo de la tobera, $\mathrm{pg}^{2}$

$\mathrm{A}_{\mathrm{S}} \quad$ Área anular de la cámara de mezclado para el flujo de la producción, pg $^{2}$

$\mathrm{A}_{\mathrm{T}} \quad$ Área de flujo total de la cámara de mezclado, $\mathrm{pg}^{2}$

D Profundidad vertical del pozo, pies

$\mathrm{D}_{1} \quad$ Diámetro interno de la tubería de producción o de la tubería de revestimiento, pg

$\mathrm{D}_{2} \quad$ Diámetro externo de la tubería interior en flujo anular, pg

$\mathrm{F}_{\mathrm{w}} \quad$ Fracción del agua de formación

$\mathrm{F}_{\mathrm{WD}} \quad$ Fracción del agua del fluido de la columna de retorno

$\mathrm{P}_{\mathrm{FN}} \quad$ Pérdida de presión por fricción del fluido motriz en la tubería de inyección, $\mathrm{lb} / \mathrm{pg}^{2}$

$\mathrm{P}_{\mathrm{FD}} \quad$ Pérdida de presión por fricción del fluido en el circuito de retorno, $\mathrm{lb} / \mathrm{pg}^{2}$

$\mathrm{G}_{\mathrm{N}} \quad$ Gradiente del fluido motriz en la tubería de inyección, $\mathrm{lb} / \mathrm{pg}^{2} / \mathrm{pie}$

$\mathrm{G}_{\mathrm{D}} \quad$ Gradiente del fluido de la columna de retorno, $\mathrm{lb} / \mathrm{pg}^{2} / \mathrm{pie}$

$\mathrm{G}_{\mathrm{S}} \quad$ Gradiente del fluido de formación, $\mathrm{lb} / \mathrm{pg}^{2} / \mathrm{pie}$

$\mathrm{G}_{\mathrm{O}} \quad$ Gradiente del petróleo producido, $\mathrm{lb} / \mathrm{pg}^{2} / \mathrm{pie}$

$\mathrm{G}_{\mathrm{W}} \quad$ Gradiente del agua de formación, $\mathrm{lb} / \mathrm{pg}^{2} / \mathrm{pie}$

$\mathrm{H} \quad$ Relación adimensional de recuperación de presión

HP Potencia, hp

$\mathrm{K}_{\mathrm{N}} \quad$ Coeficiente de pérdida en la tobera

$\mathrm{K}_{\mathrm{TD}} \quad$ Coeficiente de pérdida combinado cámara de mezclado - difusor

L Longitud de T.P. hasta la bomba $=$ profundidad de colocación de la bomba $=\mathrm{h}_{1}$, pies

M Relación de flujo adimensional, $\mathrm{q}_{3} / \mathrm{q}_{1}$

$\mathrm{M}_{\mathrm{L}} \quad$ Relación de flujo adimensional en el límite de cavitación

NUM Variable usada para definir $\mathrm{H}$

$\mathrm{P}_{\mathrm{N}} \quad$ Presión a la entrada de la tobera, $\mathrm{lb} / \mathrm{pg}^{2}$

$\mathrm{P}_{\mathrm{D}} \quad$ Presión de descarga, $\mathrm{lb} / \mathrm{pg}^{2}$

$\mathrm{P}_{\mathrm{S}} \quad$ Presión de succión, $\mathrm{lb} / \mathrm{pg}^{2}$

$\mathrm{P}_{\mathrm{T}} \quad$ Presión superficial de operación = Presión de descarga de la bomba tríplex, $\mathrm{lb} / \mathrm{pg}^{2}$

$\mathrm{P}_{\mathrm{F}} \quad$ Pérdidas de presión por fricción, $\mathrm{lb} / \mathrm{pg}^{2}$

$\mathrm{P}_{\mathrm{WH}} \quad$ Contra presión en la línea de flujo, $\mathrm{lb} / \mathrm{pg}^{2}$ 


\section{SÍMBOLO DEFINICIÓN}

$\mathrm{Q}_{\mathrm{N}} \quad$ Tasa del fluido motriz, bl/día

$\mathrm{Q}_{\mathrm{D}} \quad$ Tasa del fluido producido más fluido motriz (tasa de descarga), bl/día

Q Tasa del fluido producido, bl/día

R Relación de áreas, $A_{j} / A_{t}$

GLR Relación gas-líquido, $\mathrm{pies}^{3} / \mathrm{bl}$

GOR Relación gas-petróleo, $\mathrm{pies}^{3} / \mathrm{bl}$

T.P. Tubería de producción

$\gamma_{f} \quad$ Densidad relativa del fluido motriz (agua=1.0)

$\rho \quad$ Densidad del fluido, $\mathrm{lb} / \mathrm{pie}^{3}$

Viscosidad, cp

Viscosidad del fluido de retorno, $\mathrm{cp}$

Viscosidad del petróleo, cp

Viscosidad del agua, cp 
MELO V.

\section{BIBLIOGRAFÍA}

Brown Kermit. E.: “The Technology of Artificial Lift Methods”. Volume 2b. Petroleum Publishing Co. 1980.

Coberly, C. J., "Theory and Application of Hydraulic Oil Well Pumps”, Kobe Inc., Huntington Park, California, 1961.

Petrie Hal, Wilson Phil and Smart Eddie, "The Theory, Hardware and Application of the Current Generation of Oil Well Jet Pumps”, Southwestern Petroleum Short Course, April 27 - 28, 1983, Texas Tech University.

Petrie Hal, Wilson Phil and Smart Eddie. "Jet Pumping Oil Wells", World Oil, November and December 1983, January 1984

Smart, E.: "Jet Pump Geometry Selection", Southwestern Petroleum Short Course, April 23 - 25, 1985, Texas Tech University. 